\title{
Article
}

\section{Self-Assembling Elastin-Like Hydrogels for Timolol Delivery: Development of an Ophthalmic Formulation Against Glaucoma.}

\author{
Alicia Fernández-Colino, Daniela A. Quinteros, Daniel A. Allemandi, \\ Alessandra Girotti, Santiago D. Palma, and F. Javier Arias
}

Mol. Pharmaceutics, Just Accepted Manuscript • DOI: 10.1021/acs.molpharmaceut.7b00615 • Publication Date (Web): 10 Nov 2017

Downloaded from http://pubs.acs.org on November 13, 2017

\section{Just Accepted}

"Just Accepted" manuscripts have been peer-reviewed and accepted for publication. They are posted online prior to technical editing, formatting for publication and author proofing. The American Chemical Society provides "Just Accepted" as a free service to the research community to expedite the dissemination of scientific material as soon as possible after acceptance. "Just Accepted" manuscripts appear in full in PDF format accompanied by an HTML abstract. "Just Accepted" manuscripts have been fully peer reviewed, but should not be considered the official version of record. They are accessible to all readers and citable by the Digital Object Identifier (DOI®). "Just Accepted" is an optional service offered to authors. Therefore, the "Just Accepted" Web site may not include all articles that will be published in the journal. After a manuscript is technically edited and formatted, it will be removed from the "Just Accepted" Web site and published as an ASAP article. Note that technical editing may introduce minor changes to the manuscript text and/or graphics which could affect content, and all legal disclaimers and ethical guidelines that apply to the journal pertain. ACS cannot be held responsible for errors or consequences arising from the use of information contained in these "Just Accepted" manuscripts. 


\title{
Self-Assembling Elastin-Like Hydrogels for Timolol Delivery: Development of an Ophthalmic
}

\section{Formulation Against Glaucoma.}

\author{
Alicia Fernández-Colino, §łł Daniela A. Quinteros, \#ł Daniel A. Allemandi, \# Alessandra \\ Girotti, $\S$ Santiago D. Palma, \#* F. Javier Arias * $^{*}$ \\ \$ These authors contributed equally to this work.
}

\begin{abstract}
§Bioforge Lab, University of Valladolid, CIBER-BBN, Paseo de Belén 19, 47011 Valladolid, Spain

\#Unidad de Investigación y Desarrollo en Tecnología Farmacéutica (UNITEFA), CONICET and Departamento de Ciencias Farmacéuticas, Facultad de Ciencias Químicas, Universidad Nacional de Córdoba, Ciudad Universitaria, 5000-Córdoba, Argentina.
\end{abstract}




\begin{abstract}
This work focuses on improving the effectiveness of current therapies against glaucoma by incorporating self-assembled polymers into the ophthalmic formulation. To that end, we first studied the influence of the dispersing medium on the mechanical performance of selfassembling elastin-like (EL) and silk-elastin like (SEL) hydrogels by conducting rheological tests. These polymers were subsequently incorporated into the anti-glaucoma formulation, which contains timolol maleate (TM) as active ingredient, and in vivo tests, namely adhesion tests and intraocular pressure measurements (IOP), were performed in New Zealand rabbits. An enhanced reduction in IOP due to the presence of the polymers was observed. Moreover, differences in the effectiveness between both EL- and SEL-hydrogels, which can be explained on the basis of the different rheological properties displayed by these two systems, were also encountered. The results point to the potential of this system as a basis for the development of an ophthalmic formulation against glaucoma.
\end{abstract}

KEYWORDS. Glaucoma, Silk-elastin-like recombinamers, Elastin-like recombinamers, thermogelling, ophthalmic formulation. 


\section{INTRODUCTION}

Glaucoma is the second leading cause of blindness worldwide $^{1}$ and is a multifactorial, progressive and neurodegenerative disease characterized by atrophy of the optic nerve and loss of retinal ganglion cells that can eventually lead to loss of visual acuity and visual field. High intraocular pressure (IOP) is considered to be the greatest risk factor for the development of glaucoma, therefore most treatments involve the chronic application of eye drops containing hypotensive agents. Timolol maleate (TM) is a small, hydrophilic molecule (432 Da) which is the US Food and Drug Administration's (FDA) "gold standard" drug for the treatment of high IOP $^{2}$. Indeed, the IOP-lowering potential of this $\beta$-receptor antagonist has been reported to be between $20 \%$ and $25 \%$ from the initial values. ${ }^{3}$

Topical instillation of this and other hypotensive drugs is preferred in order to minimize systemic side effects. ${ }^{4}$ Ophthalmic drug delivery is one of the most interesting and challenging endeavors facing the pharmaceutical sector as the anatomy, physiology and biochemistry of the eye render this organ exquisitely impervious to foreign substances ${ }^{5}$. As such, most drugs are hardly absorbed, with bioavailabilities ranging from $1 \%$ to $10 \%$. Amongst other factors, such low bioavailabilities are a consequence of a rapid and extensive loss of the formulation from the pre-corneal area due to the turnover of lacrimal drainage, which decreases the residence time of the formulation on the eye surface and hampers the efficiency of this route. ${ }^{5}$ Consequently, repeated and frequent applications of topical ophthalmic formulations are usually required to achieve the desired therapeutic effect. Glaucoma treatments are usually associated with adverse reactions generated by frequent exposure of the eye to drugs and excipients. With regard to excipients, preservatives can induce ocular surface alterations that contribute to the development of secondary ophthalmic diseases, such as dry eye syndrome, which, in turn, can compromise 
patient compliance. However, the elimination of preservatives from ophthalmic formulations is not always sufficient to avoid side effects on the ocular surface. The development of topical ophthalmic formulations for the treatment of this disease therefore presents a challenge. ${ }^{6}$ As such, the incorporation of new components with beneficial properties into ophthalmic formulations that are also able to increase the bioavailability of the drug is of great interest in this field. ${ }^{7}$

The incorporation of viscosifying agents that are able to increase the residence time of the formulation in the eye is gaining increasing attention. Amongst these compounds, in situ gelforming formulations, which undergo a phase transition from a liquid to a semisolid gel upon exposure to physiological environments, are a promising approach. These formulations should be free-flowing liquids at room temperature to allow easily reproducible administration into the eye as a drop. They should also undergo an in situ phase transition to form a gel that is able to withstand shear forces in the cul de sac and of sustaining drug release under physiological conditions ${ }^{8}$. As such, biomaterials science is fast becoming a cornerstone to help to meet the therapeutic challenges faced by ophthalmologists when treating glaucoma. ${ }^{6,9}$

An excellent approach to in situ hydrogel formation takes advantage of the self-assembling nature displayed by some protein-based polymers. ${ }^{10}$ Many self-assembling motifs have been explored ${ }^{11,12}$ in materials science, with those inspired by the sequence of elastin ${ }^{13}$ (elastin-like polymers, ELPs), silk ${ }^{14}$ (silk-like polymers, SLPs) or by a combination of the two (silk elastin like polymers, SELPs) being especially relevant. Furthermore, in order to ensure strict control over the sequence, chain complexity and monodispersity, recombinant DNA technology has been implemented to bio-produce this class of materials, therefore a new term, namely 
recombinamer, has been established to refer to the polymers produced using recombinant technology. $^{15,16}$

The ELP or their recombinant counterparts, known as elastin-like recombinamers (ELR) ${ }^{17}$, are artificial polypeptides that are bioinspired in the natural elastin. The native elastin is an insoluble protein formed by the interaction of various molecules of tropoelastin (its soluble precursor). Tropoelastin is composed of two different types of domains, namely crosslinking domains and elastomeric structural domains, which are formed by repeating sequences as poly (VPG), poly (VPGG), poly (GVGVP), poly (IPGVG), poly (VAPGVG), where V stands for valine, $\mathrm{P}$ for proline $\mathrm{G}$ for glycine, A for alanine and I for isoleucine. The sequences of the elastin-based artificial polymers mimic these patters, and the vast majority have the formula (VPGXG) $)_{\mathrm{n}}$, where " $n$ " is the number of repetitions and " $\mathrm{X}$ " represents any amino acid except proline. An amphiphilic ELR tetrablock copolymer from this family, which contains two different kinds of blocks, and was developed to generate micro-patterned biocompatible hydrogels, has been reported previously ${ }^{18}$. The hydrophobic blocks, which follow the patterned poly (IPGVG), are responsible for physical cross-linking of the hydrogel (by means of hydrophobic contacts) when the system reaches body temperature due to the characteristic inverse temperature transition $(\text { ITT })^{19}$ experienced by these materials. Briefly, this transition involves the conformational change of the hydrophobic elastin-like domain from a soluble, random state at low temperature to an aggregated state characterized by a succession of $\beta$-turns above a specific temperature ${ }^{20,21}$ known as the transition temperature $(\mathrm{Tt})$. The remaining two blocks of the molecule are characterized by the presence of VPGEG pentapeptides, where E stands for glutamic amino acid. The amino acid glutamic displays a carboxylate group which is ionized at neutral $\mathrm{pH}$ and therefore it provides a hydrophilic character. Therefore, VPGEG-containing blocks are a random, 
soluble state under physiological conditions $\left(37^{\circ} \mathrm{C}\right.$, neutral $\left.\mathrm{pH}\right),{ }^{22}$ and exert a water-retention function required for an hydrogel state.

Silk-like recombinamers are bioinspired by the sequence of silk. ${ }^{23}$ One of the most popular motifs is the hexapeptide GAGAGS (where A and S stand for the amino acids alanine and serine respectively), which is naturally present in the heavy chain of silk fibroin produced by the worm Bombyx mori. ${ }^{24}$ The interest in this domain is due to its ability to mediate irreversible and stable physical interactions by adopting a $\beta$-sheet conformation. In this respect, the combination of both kinds of domains has given rise to the so-called silk-elastin like recombinamers. (EIS)x2, which belongs to this family, is constituted by a combination of EL blocks and SL blocks, with the former being found in a tetrablock, thermally triggered, amphiphilic molecule, equivalent to that of (EI)x2 (Figure 1). This material self-organizes from a sol state to a stable fibrous gel state. ${ }^{22}$ In (EIS)x2, EL blocks clearly dominate the final structure compared to other SELRs found in the literature. The small proportion of SL blocks to EL blocks used in this construct allows to maintain the self-assembling properties of the EL-blocks.

a) ELR tetrablock copolymer: (EI)x2

\begin{tabular}{|c|c|c|c|}
\hline E & I & E & I \\
\hline Hydrophilic EL-block & Hydrophobic EL-block & Hydrophilic EL-block & Hydrophobic EL-block
\end{tabular}

b) SELR copolymer: (EIS) $\times 2$

\begin{tabular}{|c|c|c|c|c|c|}
\hline E & I & S & E & I & SL-block \\
\hline Hydrophilic EL-block & Hydrophobic EL-block & Hydrophilic EL-block & Hydrophobic EL-block
\end{tabular}

Figure 1: Schematic diagram showing the different domains of the recombinamers (EI)x2 and $(E I S) \times 2$. 
Self-assembly processes of protein-based polymers (e.g. ELR and SELR) have been reported to be influenced by environmental conditions such as $\mathrm{pH}$, temperature and sonication, ${ }^{25}$ and this feature has been exploited to create devices that are able to sense surrounding stimuli. ${ }^{26}$ Similarly, this fact opens the door to further tuning the properties of a given material by controlling the external inputs to which it is exposed. With regard to ELR self-assembly, it has been reported that the composition of the dispersing medium plays a pivotal role in determining the nanometer-size features of the resulting micelle-like ELR nanoparticles. ${ }^{27,}{ }^{28}$ However, many of these studies have focused on the change of properties on a nano-scale, and little attention has been paid to the possible effects on macroscale performance.

In light of the above, this work focuses on determining the influence of such variables on the physical properties of the hydrogel, as well as the translation of such fundamental studies to the practical aim of developing an ophthalmic anti-glaucoma formulation. Thus, four different types of aqueous solutions, namely deionized water, glucose 5\%, $\mathrm{NaCl} 0.9 \%$ and $\mathrm{PBS}$ (phosphate buffered saline), were selected according to their relevance for their envisaged biomedical application, $\mathrm{PBS}$ and $\mathrm{NaCl} 0.9 \%$ and glucose $5 \%$ are highly used solutions in biomedical research and clinics, and the three of them provide a osmolarity equivalent to that found in physiological conditions (270-330 mOsmol/L). Deionized water was included as the control dispersing medium, characterized by the absence of solutes. In situ gelation behavior in these solutions was studied.

Furthermore, the use of both recombinamers as components of an ophthalmic formulation against glaucoma was also evaluated by performing in vivo irritation tests, adhesion tests and IOP measurements in New Zealand rabbits. 


\section{EXPERIMENTAL SECTION}

\subsection{Materials}

TM was supplied by Parafarm (Buenos Aires, Argentina) and sodium chloride by Cicarelli Reagents (Rosario, Argentina). Glucose 5\% was supplied by Roux Ocefa (Buenos Aires, Argentina), and (EI)x2 and (EIS)x2 were produced by recombinant technology and purified by Bioforge laboratory as reported elsewhere. ${ }^{15,18,22}$ The nomenclature used for referring to each block contained in each recombinamer is provided in Table 1 and the amino acid sequences of the different recombinamers is shown in Table 2

Table 1: Correspondence of the abbreviations used to name each block that composes each recombinamer.

\begin{tabular}{|c|c|c|}
\hline Block & Amino acidic sequence & Source of \\
\hline $\mathbf{E}$ & {$\left[(\mathrm{VPGVG})_{2}-(\mathrm{VPGEG})-(\mathrm{VPGVG})_{2}\right]_{10}$} & Elastin \\
\hline $\mathbf{I}$ & {$[\mathrm{VGIPG}]_{60}$} & Elastin \\
\hline $\mathbf{S}$ & {$\left[(\mathrm{GAGAGS})_{5} \mathrm{G}\right]_{2}$} & Silk \\
\hline
\end{tabular}

Table 2. Amino acid sequence of each recombinamer

\begin{tabular}{|c|l|}
\hline $\begin{array}{c}\text { Abbreviated } \\
\text { name }\end{array}$ & \multicolumn{1}{c|}{ Amino acid sequence } \\
\hline (EI)x2 & MESLLP- $\{[(\mathrm{VPGVG}) 2-(\mathrm{VPGEG})-(\mathrm{VPGVG}) 2] 10[\mathrm{VGIPG}] 60\} 2-\mathrm{V}$ \\
\hline (EIS)x2 & $\begin{array}{l}\text { MESLLP- }\{[(\mathrm{VPGVG}) 2-(\mathrm{VPGEG})-(\mathrm{VPGVG}) 2] 10[\mathrm{VGIPG}] 60)- \\
[\mathrm{V}(\mathrm{GAGAGS}) 5 \mathrm{G}] 2\} 2-\mathrm{V}\end{array}$ \\
\hline
\end{tabular}


Deionized water was used in all experiments. PBS (phosphate buffered saline: $\mathrm{KH}_{2} \mathrm{PO}_{4}$ $0.0144 \%(\mathrm{w} / \mathrm{v}) ; \mathrm{NaCl} 0.9 \%(\mathrm{w} / \mathrm{v})$ and $\mathrm{Na}_{2} \mathrm{HPO}_{4}$ 0.0795\% (w/v), $\left.\mathrm{pH} 7.4\right)$ and $\mathrm{NaCl}$ were of analytical grade and were used without further purification.

\subsection{Production of (EI)x2 and (EIS) $x 2$}

The gene sequences encoding for (EI)x2 and (EIS)x2 were available in the laboratory from previous studies $^{18,22}$ and had been constructed through standard molecular biologic techniques. Specifically, we used the iterative- recursive directional ligation (RDL) method ${ }^{29}$, which allows controlled and sequential concatenation of the gene segments, resulting in a multiblock-coding gene. The multiblock-coding genes sequences encoding for (EI)x2 and (EIS)x2 were subcloned into a modified version of pET-25(+) expression vector. Finally, they were transformed into the E. coli strain BL21 Star (DE3) (Invitrogen) for subsequent expression and production of the recombinamers. The purification protocol consisted of sequential rounds of inverse transition cycling (ITC). The purity and molecular weight of the recombinamers were routinely determined by sodium dodecyl sulfate polyacrylamide gel electrophoresis (SDS-PAGE), NMR (nuclear magnetic resonance) analysis amino acid analysis and mass spectrometry (MALDI-TOF/MS).

\subsection{Rheology tests}

The mechanical properties of (EI)x2 and (EIS)x2 hydrogels were measured in a controlled stress rheometer (AR2000ex, TA Instruments) equipped with a Peltier plate temperature control. Thus, $350 \mu \mathrm{L}$ of each recombinamer solution at $15 \mathrm{wt} . \%$ in the corresponding dispersing medium 
(deionized water, glucose $5 \%, \mathrm{NaCl} 0.9 \%$ and $\mathrm{PBS}$ ) were placed on the Peltier plate of the rheometer pre-cooled to $5^{\circ} \mathrm{C}$. A parallel plate geometry with a diameter of $20 \mathrm{~mm}$ was used. Temperature ramp experiments were performed by heating the sample from 5 to $37{ }^{\circ} \mathrm{C}$. The heating rate was $2.5^{\circ} \mathrm{C} / \mathrm{min}$, and the reverse process (cooling) was performed under the same conditions. A constant strain of $0.5 \%$ (within the linear viscoelastic region) and a frequency of $10 \mathrm{~Hz}$ were used.

\subsection{Differential scanning calorimetry (DSC)}

DSC experimentswere performed using a Mettler Toledo 822e with liquid-nitrogencooler. Both temperature and enthalpy were calibrated using a standard indiumsample. (EI)x2 and (EIS)x2 samples for the DSC measurements wereprepared at 15 wt.\% in PBS, $\mathrm{NaCl} 0.9 \%$, deionized water and glucose $5 \%$.A volume of $20 \mu \mathrm{L}$ of the corresponding sample was placed inside astandard $40 \mu \mathrm{L}$ aluminum pan and sealed hermetically. The heating program for DSC measurements included an initial isothermal step $\left(5 \mathrm{~min}\right.$ at $0{ }^{\circ} \mathrm{C}$ to stabilize the temperature and the state of the tetrablock), followed by heating from $0{ }^{\circ} \mathrm{C}$ to $60^{\circ} \mathrm{C}$ at $5{ }^{\circ} \mathrm{C} / \mathrm{min}$.

\subsection{In vitro erosion testing of the recombinamers}

(EI)x2 and (EIS)x2 solutions at $15 \mathrm{wt} . \%(1 \mathrm{ml})$ were prepared in dextrose 5\% and kept at 35 ${ }^{\circ} \mathrm{C}$ to achieve a gel state. Then, $3.5 \mathrm{ml}$ of buffer solution $\mathrm{pH} 6.8$ was added with gentle shaking at $50 \mathrm{rpm}$. At fixed times, $250 \mu \mathrm{L}$ samples were removed and replaced by fresh buffer. The erosion of the hydrogels was determined using Biuret reagent $\left(\mathrm{CuSO}_{4}\right.$ at $2 \%+\mathrm{NaOH}$ at $\left.40 \%\right)$. The 
concentration of (EI)x2 and (EIS) 2 in the released medium was determined by UV spectrophotometry at a maximum absorbance wavelength of $540 \mathrm{~nm}$ (UV VIS TERMO Evolution 300). All experiments were performed in triplicate.

\subsection{In vitro drug-release studies}

Solutions of (EI)x2 and (EIS)x2 (15 wt.\%) containing TM (0.5 w/v.\%) (1 ml) were prepared and subsequently kept at $35{ }^{\circ} \mathrm{C}$ for $10 \mathrm{~min}$ to ensure hydrogel formation. Thereafter, $3.5 \mathrm{ml}$ of buffer solution pH 6.8 was added with gentle shaking at $50 \mathrm{rpm}$. At appropriate intervals, $250 \mu \mathrm{L}$ samples were removed and replaced with fresh buffer. The quantity of TM in the release medium was determined by high-performance liquid chromatography (HPLC, see section 2.6). Each sample was assayed in triplicate $(n=3)$. Mathematical analysis was performed by adjusting the experimental values to the Korsmeyer-Peppas model.

$$
\frac{M_{t}}{M_{\infty}}=k \cdot t^{n}
$$

In the above, "Mt" is the cumulative amount of drug released at time "t", "Mळ" is the cumulative amount of drug released at infinite time, " $k$ " is a rate constant incorporating characteristics of the macromolecular network of the system and the drug, and " $n$ " is the release exponent, which is related to the mechanism of drug release. If $\mathrm{n}=0.5$, the release is governed by Fickian diffusion, whereas $n=1$ indicates that molecules are released by surface erosion; both mechanisms play a role in release for " $\mathrm{n}$ " values between 0.5 and $1 .{ }^{30}$ 


\subsection{HPLC determinations of TM}

The HPLC system consisted of a Waters ${ }^{\circledR}$ HPLC pump and a Waters ${ }^{\circledR}$ HPLC detector set at $295 \mathrm{~nm}$. Samples were chromatographed on a reversed-phase Luna C18 column (250 x $4.6 \mathrm{~mm}$, $5 \mathrm{~mm}$, Phenomenex) and a $2 \times 8 \mathrm{~mm}$ pre-column of the same material, with the mobile phase having a flow rate of $1 \mathrm{~mL} / \mathrm{min}$ and consisting of trifluoroacetic acid $0.05 \%(\mathrm{v} / \mathrm{v})$ in acetonitrile (40:60, v/v), which was filtered and degassed before use. The column was thermostated at $25^{\circ} \mathrm{C}$.

\subsection{Cytocompatibility}

The HFF-1 (human foreskin fibroblast) cell line was used as cell model to test the cytocompatibility of the recombinamers. Thus, 7500 HFF-1 cells were seeded onto 96-well culture plates, then the culture medium was removed after $5 \mathrm{~h}$ and replaced with $100 \mu \mathrm{L}$ of the corresponding recombinamer solution at $25 \mu \mathrm{M}$ in culture medium (DMEN). A $100 \mu \mathrm{L}$ aliquot of DMEN (with no recombinamer) was added in the case of the negative controls. Live and dead staining (LIVE/DEAD Viability/Cytoxicity Assay Kit, Invitrogen) was used according to the manufacturer's instructions, and fluorescence intensity emission was measured at 425 and 620 $\mathrm{nm}$ after excitation at 485 and $525 \mathrm{~nm}$ (SpectraMax M5e microplate reader, Molecular Devices), respectively, after culture for $24 \mathrm{~h}$. The number of live and dead HFF-1 cells was calculated from the fluorescence intensity using a calibration curve obtained with known numbers of HFF-1 cells seeded on 96-well plates (from 1000 to 20,000 cells per mL, using $100 \mu \mathrm{L}$ of DMEN medium). Statistical analysis was performed by One Way Analysis of Variance (ANOVA). Images of the cells after Live and dead staining were taken with a Nikon Eclipse Ti-SR (Japan) fluorescence 
microscope. Three independent experiments, each in triplicate, were performed for each recombinamer.

\subsection{In vivo mucoadhesion tests}

Solutions of each recombinamer at $15 \mathrm{wt} \%$ with sodium fluorescein at $0.25 \%$ in dextrose solution at $5 \%$ were prepared, and $50 \mu \mathrm{L}$ of the corresponding solution was placed in the inferior conjunctival fornix of the right eyes of three rabbits. The left eyes were used as controls and were treated with $50 \mu \mathrm{L}$ of commercial fluorescein at $0.25 \%$.

The behavior of each solution in terms of residence and adherence on the eye surface was evaluated using a binocular indirect ophthalmoscope (Neitz IO- small pupils, Tokyo, Japan) and 20 diopter lens (Nikon, Tokyo, Japan) and a score was calculated for each time point according to the parameters presented in Table 3. A one-way ANOVA statistical analysis was performed and the Holm-Sidak method was applied.

Table 3: Proposed score rating for evaluating bioadhesion intensity in vivo. The presence of the formulation is assessed by inspecting several regions and tissues in the eye, and a score is assigned according to the observed appearance. A total score encompassing the global behavior of the formulation is obtained by summing the scores obtained for each region.

\begin{tabular}{|c|c|c|}
\hline TISSUE/REGION & APPEARANCE & SCORE \\
\hline \multirow{4}{*}{ CORNEA } & Complete & 4 \\
\cline { 2 - 3 } & $3 / 4$ Cornea & 3 \\
\cline { 2 - 3 } & $1 / 2$ Cornea & 2 \\
\cline { 2 - 3 } & $1 / 4$ Cornea & 1 \\
\hline
\end{tabular}




\begin{tabular}{|c|c|c|}
\hline \multirow{4}{*}{ CONJUNTIVAL SAC } & Nothing & 0 \\
\cline { 2 - 3 } & Abundant & 3 \\
\cline { 2 - 3 } & Medium & 2 \\
\cline { 2 - 3 } & Scarce & 1 \\
\hline \multirow{3}{*}{$\begin{array}{c}\text { MACHRYMAL } \\
\text { MENISCUS }\end{array}$} & Nothing & 0 \\
\cline { 2 - 3 } & $2 \mathrm{~mm}$ & 3 \\
\cline { 2 - 3 } & $1 \mathrm{~mm}$ & 2 \\
\hline \multirow{2}{*}{ EYELID } & Thin line & 1 \\
\cline { 2 - 3 } & Nothing & 0 \\
\hline \multirow{2}{*}{ NOSE } & Wet & 1 \\
\hline & Notwet & 0 \\
\hline
\end{tabular}

\subsection{In vivo study of the hypotensive efficacy: IOP determinations}

Experiments were performed in both eyes of non-sedated normotensive male New Zealand white rabbits $(2-2.5 \mathrm{~kg})$. The animals were kept in individual cages with free access to food and water and maintained in a controlled 12/12 h light/dark cycle. Animal management procedures conformed to the ARVO (Association for Research in Vision and Ophthalmology) resolution on the use of animals in research, the European Communities Council Directive (86/609/EEC), and the Institutional Care and Use Committee of the Chemistry Faculty of Córdoba University (Córdoba, Argentina) reviewed and approved the protocols.

The corresponding recombinamer, namely (EI)x2 or (EIS)x2, was dissolved at 15 wt.\% in glucose $5 \%(\mathrm{w} / \mathrm{v})$ solution with $0.5 \% \mathrm{TM}$ (Parafarm), then $50 \mu \mathrm{L}$ of each formulation was placed into the conjunctival fornix of the eye of the rabbits $(n=15$ for each formulation). As controls for 
the recombinamer effect, additional rabbits $(n=20)$ were treated with glucose $5 \%(\mathrm{w} / \mathrm{v})$ solution with TM $0.5 \%$ (with no recombinamer). In order to establish the basal IOP of the animals, additional eyes $(\mathrm{n}=18)$ were treated with glucose $5 \%(\mathrm{w} / \mathrm{v})$ solution alone (with no recombinamer or TM). IOP was measured using a Tonovet rebound tonometer (Tiolat, Helsinki, Finland), which allows IOP to be assessed without the need for topical anesthesia. For each eye, IOP was set at $100 \%$ with two basal readings taken $30 \mathrm{~min}$ before and immediately before the instillation. The evolution of the IOP was measured each hour, for a period of up to $8 \mathrm{~h}$.

\subsection{In vivo ocular irritation test}

The potential ocular irritancy and/or damaging effects of the samples tested were evaluated using a slightly modified version of the Draize test. ${ }^{31}$ Thus, for each recombinamer sample, a test was carried out on three New Zealand rabbits using a volume of $50 \mu \mathrm{L}$ of recombinamer solution at 15 wt. $\%$ in dextrose $5 \%$ with $\mathrm{TM}$ at $0.5 \%$. This solution was placed in the conjunctival fornix of the right eyes, with the left eyes being used as negative control. The commercial solution Zopirol $0.5 \%$ TM (Elea Visual) was used as the negative irritation control. Post-exposure evaluations of the conjunctiva, cornea and iris were performed by external observation under adequate illumination, and additional information was obtained using a binocular indirect ophthalmoscope (Neitz IO- small pupils Tokyo, Japan) and 20 diopter lens (Nikon, Tokyo, Japan). For each observation, one drop of fluorescein salt $(0.25 \%)$ was instilled to contrast the potential corneal injury. The ocular irritation or damage was scored by successive inspections at 0, 30, 60, 90 and 120 min according to the outcomes listed in Table 4 a), and the total score for each formulation at each time point was calculated by summing the scores obtained for each region. The effects of the formulation in terms of irritation, namely no irritation or mild, 
moderate, or severe irritation, were established by comparing the total score obtained for each condition with Table 4 b).

Table 4: Evaluation parameters for ocular irritation. a) Scoring proposed for Regulatory Agencies to evaluate in vivo ocular irritation. The total score is calculated from the sum of all scores obtained for the cornea, iris and conjunctivae. Adapted from ${ }^{31,32}$. b) Formulation effects corresponding to each score value.

a)

\begin{tabular}{|c|c|c|}
\hline Region & Symptoms & Score \\
\hline \multirow{5}{*}{$\begin{array}{l}\text { Corneal } \\
\text { opacity } \\
\text { (most dense } \\
\text { area taken for } \\
\text { reading) }\end{array}$} & No opacity or keratitis. & 0 \\
\hline & Opacity or diffuse keratitis, details of iris clearly visible. & 1 \\
\hline & Easy discernible translucent area, details of iris slightly obscured. & 2 \\
\hline & $\begin{array}{l}\text { Opalescent regions; no details of iris visible, size of pupil barely } \\
\text { discernible. }\end{array}$ & 3 \\
\hline & Opaque cornea; iris not discernible through the opacity. & 4 \\
\hline \multirow{4}{*}{ Iritis } & Normal. & 0 \\
\hline & Turbidity in the aqueous humor & 2 \\
\hline & $\begin{array}{l}\text { Deepening of iris rugae and/or iris congestion or swelling, with } \\
\text { perikeratic injection. }\end{array}$ & 4 \\
\hline & Hemorrhage, gross destruction of iris or nonreactivity to light & 6 \\
\hline \multirow{5}{*}{$\begin{array}{l}\text { Conjunctival } \\
\text { redness }\end{array}$} & Normal blood vessel. & 0 \\
\hline & Some blood vessels definitely hyperemic (injected) & 1 \\
\hline & Diffuse crimson color; individual vessels not easily discernible. & 2 \\
\hline & Diffuse dark red & 3 \\
\hline & Chemosis & 4 \\
\hline
\end{tabular}


b)

\begin{tabular}{|c|c|}
\hline Total score & Formulation effects \\
\hline $0-1$ & Not irritating \\
\hline $2-6$ & Mild irritation \\
\hline $7-11$ & Moderate irritation \\
\hline $12-14$ & Severe irritation \\
\hline
\end{tabular}

1

2

5

6

7

8

9

10

11

12

13

14

15

16

17

18

19

20

21

22

23

24

25

26

27

28

29

30

31

32

33

34

35

36

37

38

39

40

41

42

43

44

45

46

47

48

49

50

51

52

53

54

55

56

57

58

59

60

ACS Paragon Plus Environment 


\section{RESULTS AND DISCUSSION}

\subsection{Rheology and DSC}

Rheological measurements of (EI)x2 and (EIS)x2 at 15 wt.\% in the four different types of dispersing media under study, namely $\mathrm{PBS}, \mathrm{NaCl} 0.9 \%$, glucose $5 \%$ and deionized water, all of them at physiological $\mathrm{pH}$, were performed in order to check the possible influence of the composition of the different media on the mechanical performance.

Figure 2 shows the recorded $\mathrm{G}^{\prime}$ (elastic or storage modulus) and $\mathrm{G}^{\prime \prime}$ (viscous or loss modulus). The gel point is usually considered as the point from which the storage modulus surpasses the loss modulus, which indicates that the sample has transitioned from fluid-like behavior to viscoelastic solid behavior. Therefore, the gelation temperature can be determined as the crossover temperature between $\mathrm{G}^{\prime}-\mathrm{G}^{\prime \prime 3}$. As shown in Figure 2, at low temperatures, all the samples

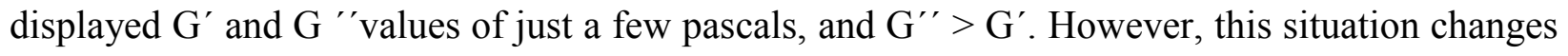
for all eight samples (both recombinamers, each in four different types of dispersing media) in the temperature range $10-22{ }^{\circ} \mathrm{C}$, in which $\mathrm{G}^{\prime}$ surpasses $\mathrm{G}^{\prime \prime}$, indicating gel formation (Table 5), as 34. Additionally, DSC experiments were performed in order to check the $\mathrm{Tt}$ of the recombinamers (Supplementary data, Figure S1). It is worth noting that the resulting values are close to the temperature gelation values measured by rheology (Table 5). This similarity is due to the gelation mechanism that governs these materials, in which EL moieties undergo conformational changes from an extended state (below the $\mathrm{Tt}$ ) to a folded one (above the $\mathrm{Tt}$ ). This folded state involves the establishment of hydrophobic contacts, which result in gel formation. For the four conditions tested, (EIS)x2 displayed a slightly higher gelling temperature (between 1 and $2{ }^{\circ} \mathrm{C}$ higher) than (EI)x2 under the same conditions (Table 5). The presence of the hydrophilic amino 
acid serine in the sequence of the SL motif could be responsible for the observed increase in the gelation temperature for (EIS)x2 with respect to (EI)x2.

As regards (EI)x2, the gel state was not stable over the whole range of temperatures measured when the dispersing media were $\mathrm{NaCl} 0.9 \%$ or PBS (Figure 2 a and c). Although a gel state was clearly present at $20^{\circ} \mathrm{C}$, the mechanical performance of the material was markedly decreased at physiological temperature $\left(37^{\circ} \mathrm{C}\right)$. In other words, the gel state was close to disappear for the sample with PBS (Figure 2 a) and was almost negligible in the sample with $\mathrm{NaCl} 0.9 \%$ (Figure 2 c).It is remarkable that this behavior contrasts with the behavior experienced by the system when the dispersing media were deionized water or glucose $5 \%$, in which there were no signs of instability and the gel states were maintained at $37{ }^{\circ} \mathrm{C}$ (the value of $\mathrm{G}^{\prime}$ was clearly higher than $\mathrm{G}^{\prime \prime}$; Figure 2 e and g, and Table 5). This behavior is also patently clear by looking at the $\tan \delta$ values. Tan $\delta$ represents the ratio of viscous modulus $\left(\mathrm{G}^{\prime \prime}\right)$ to elastic modulus $\left(\mathrm{G}^{\prime}\right)$, and it is therefore a quantifier of the dominance of the elastic or the viscous behavior. As shown in Table $3, \tan \delta$ values were higher when the dispersing medium was PBS or $\mathrm{NaCl}$ than when glucose $5 \%$ or deionized water were used, indicating a more viscous and less elastic behavior of the former.

A similar tendency to that observed for (EI)x2 was found for (EIS)x2, namely the presence of $\mathrm{NaCl} 0.9 \%$ and PBS provoked certain instability in the gel state at $37^{\circ} \mathrm{C}$. However, in this case, this instability did not result in the disappearance of the gel state, as $\mathrm{G}^{\prime}$ was clearly higher than $\mathrm{G}^{\prime \prime}$ under these conditions (Figure 2). The slight reduction in the mechanical properties of the gel state observed upon warming from 20 to $37^{\circ} \mathrm{C}$ when the dispersing medium was $\mathrm{NaCl} 0.9 \%$ or PBS was not encountered when the medium was deionized water or glucose $5 \%$. 

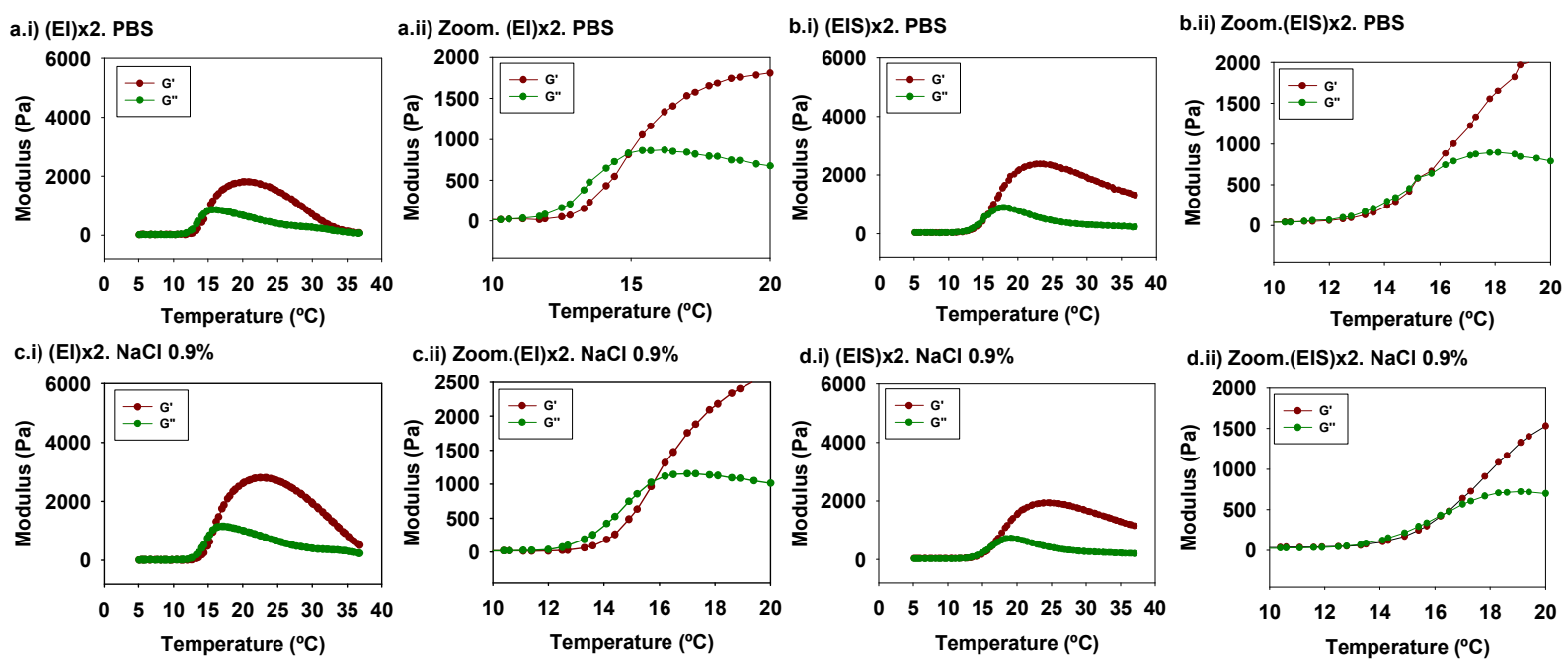

d.i) (EIS) $\times 2 . \mathrm{NaCl} 0.9 \%$

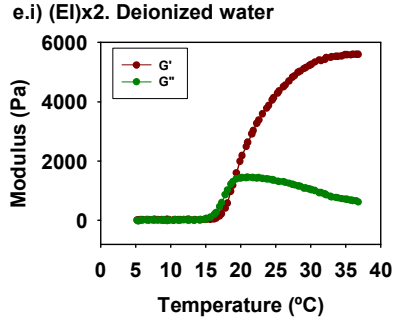

e.ii) Zoom.(EI)x2. Deionized water
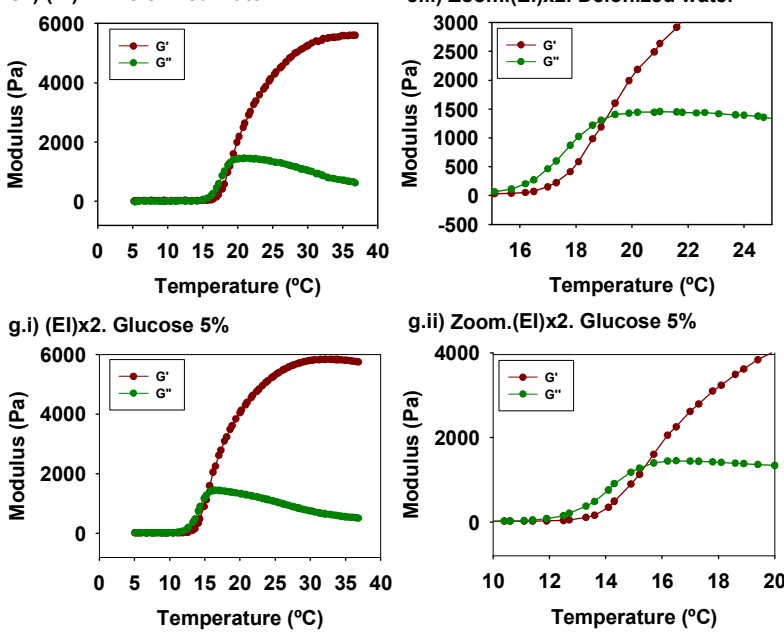

g.ii) Zoom.(El)x2. Glucose $5 \%$
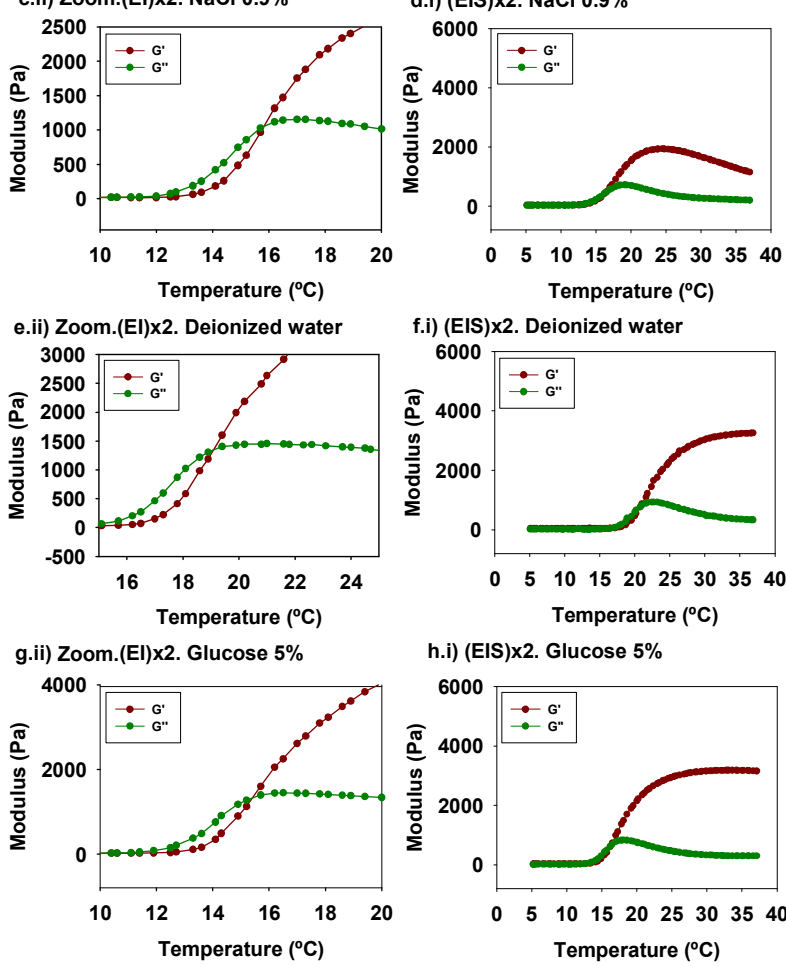

d.ii) Zoom.(EIS)x2. $\mathrm{NaCl} 0.9 \%$
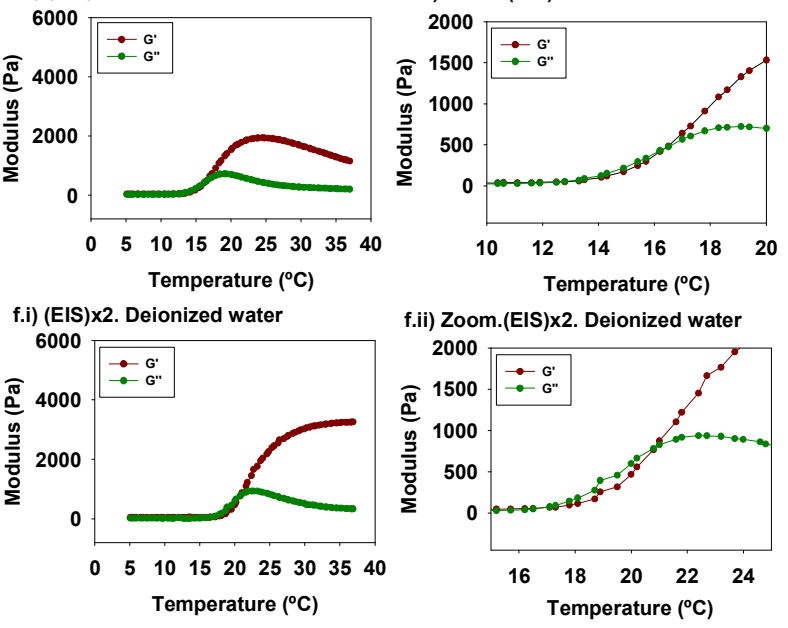

h.i) (EIS)x2. Glucose 5\%

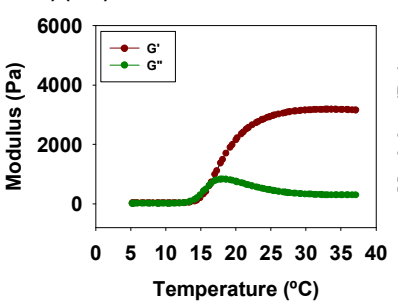

Figure 2: Storage $\left(G^{\prime}\right)$ and loss moduli ( $\left.G^{\prime \prime}\right)$ for (EI)x2 (left) and (EIS)x2 (right) recombinamer solutions (15 wt.\%) as a function of temperature in different dispersing media. Measurements were performed at $10 \mathrm{~Hz}$. a) and b) $\mathrm{PBS}$. c) and d) $\mathrm{NaCl} \mathrm{0.9 \% .} \mathrm{e)} \mathrm{and} \mathrm{f)} \mathrm{deionized}$ water. g) and h) glucose $5 \%$.

Table 5: I) Compilation of the temperatures at which the cross-over between $G^{\prime}$ and $G^{\prime \prime}$ takes place for (EI)x2 and (EIS)x2 in the different dispersing media, and the concomitant complex 
modulus $\left(G^{*}\right)$ obtained at $37{ }^{\circ} \mathrm{C}$ for each recombinamer. II) Further detailed mechanical properties $\left(G^{\prime}, G^{\prime \prime}\right.$ and tan $\left.\delta\right)$ displayed by (EI)x2 and (EIS)x2 in different dispersing media at $37^{\circ} \mathrm{C}$

I)

\begin{tabular}{|c|c|c|c|c|}
\hline & \multicolumn{2}{|c|}{$\begin{array}{c}G^{\prime}-G^{\prime \prime} \text { crossover } \\
\left({ }^{\circ} \mathbf{C}\right)\end{array}$} & \multicolumn{2}{|c|}{$\begin{array}{c}\mathrm{G}^{*}\left(37^{\circ} \mathrm{C}\right) \\
(\mathrm{Pa})\end{array}$} \\
\hline & $(E I) \times 2$ & (EIS)x2 & (EI) $\times 2$ & $($ EIS) $x 2$ \\
\hline PBS & 18.9 & 20.8 & 105 & 1340 \\
\hline $\mathrm{NaCl} 0.9 \%$ & 15.7 & 16.5 & 570 & 1200 \\
\hline Deionized & 15.7 & 17.0 & 5630 & 3200 \\
\hline Glucose 5\% & 14.9 & 15.7 & 5760 & 3180 \\
\hline
\end{tabular}

II)

\begin{tabular}{|c|c|c|c|c|c|c|}
\hline & \multicolumn{3}{|c|}{$(E I) \times 2$} & \multicolumn{3}{|c|}{ (EIS)x2 } \\
\hline & $\mathrm{G}^{\prime}(\mathrm{Pa})$ & $\mathrm{G}^{\prime \prime}(\mathrm{Pa})$ & $\tan \delta$ & $\mathrm{G}^{\prime}(\mathrm{Pa})$ & $\mathrm{G}^{\prime \prime}(\mathrm{Pa})$ & $\tan \delta$ \\
\hline PBS & 84 & 65 & 0.77 & 1320 & 237 & 0.18 \\
\hline $\mathrm{NaCl} 0.9 \%$ & 520 & 340 & 0.65 & 1150 & 202 & 0.18 \\
\hline Deionized & 5600 & 623 & 0.11 & 3190 & 331 & 0.10 \\
\hline Glucose $5 \%$ & 5740 & 515 & 0.09 & 3160 & 300 & 0.10 \\
\hline
\end{tabular}

In summary, the presence of $\mathrm{NaCl} 0.9 \%$ or PBS exerts a destabilizing effect on the maintenance of the gel state at $37^{\circ} \mathrm{C}$ of both (EI)x2 and (EIS)x2 samples. This destabilization is more prominent for (EI)x2 than for (EIS)x2 due to the presence of the SL block in the latter, which is able to improve the mechanical performance. ${ }^{22}$

Furthermore, no signs of instability or a reduction in the mechanical performance of (EI)x2 and (EIS)x 2 when changing the temperature of the samples from 20 to $37^{\circ} \mathrm{C}$ are observed when the dispersing medium is deionized water or glucose $5 \%$. Taken together, these findings point to an influence of the composition of the dispersing medium on the hydrophobic contacts mediated by the EL-blocks. Such blocks are present in both recombinamers and are responsible for triggering 
the thermo-gelling process of both systems. When the composition of the dispersing media is analyzed, it can be deduced that the presence of $\mathrm{NaCl}$ plays an important role in this destabilization as it is the only component present in both $\mathrm{PBS}$ and $\mathrm{NaCl} 0.9 \%$ solutions, and at the same concentration. The destabilization of the gel state exerted by both media is similar, and the $\tan \delta$ values (Table 5, II) clearly point to a decrease in the elastic behavior in both dispersing media (PBS and $\mathrm{NaCl} 0.9 \%$ ). However, the remaining components of $\mathrm{PBS}$ (i.e. $\mathrm{KH}_{2} \mathrm{PO}_{4}$ and $\mathrm{Na}_{2} \mathrm{HPO}_{4}$ ) seem to accentuate the destabilization of the EL block-mediated gelation to a small extent as the complex modulus (i.e. the overall resistance to deformation, that encompasses both the elastic and the viscous moduli) for (EI)x2 in PBS is slightly lower, and tan $\delta$ is higher, than in $\mathrm{NaCl} 0.9 \%$ (Table 5). Moreover, the concentration of $\mathrm{KH}_{2} \mathrm{PO}_{4}$ and $\mathrm{Na}_{2} \mathrm{HPO}_{4}$ in $\mathrm{PBS}$ is about 10 times lower than the $\mathrm{NaCl}$ concentration, which could explain the almost negligible effect of these compounds on the stability when compared to the marked effect of $\mathrm{NaCl}$.

It is also noticeable that the modulus displayed by (EI)x2 in a dispersing medium lacking salts, namely deionized water or glucose $5 \%$ (Figure 2 e and g), was higher than that displayed by (EIS)x2 (Figure $2 \mathrm{f}$ and $\mathrm{h}$ ). This effect could be explained by the lower amount of EL moieties per molecule in relative terms when comparing (EIS)x2 to (EI)x2. Thus, EL moieties constitute $100 \%$ of (EI)x 2 molecules but only $90 \%$ in the case of (EIS)x2. Importantly, neither of these hydrogels was destabilized upon changing the temperature from 20 to $37^{\circ} \mathrm{C}$ in these dispersing media.

In light of the above, it is clear that dispersing media exert an influence on the mechanical properties of both systems, although the underlying mechanism responsible for the different responses remains unclear. Numerous studies have reported the influence of salts on the ITT behavior of ELRs, and it is well known that salts cause a concentration-dependent decrease in $T_{t}$ 
and an increase in the transition enthalpy ${ }^{27,35}$. With regard to the nanostructured properties of ELR, $\mathrm{NaCl}$ has been reported to have an influence on the size and shape of the resulting nanoobjects ${ }^{27}$. However, to the best of our knowledge, this is the first time that the influence of salts on the macroscale behavior of an elastin-based material, namely the mechanical performance, has been reported. Diblockcopolypeptide amphiphilic polymers containing charged and hydrophobic segments have been reported to be weakened by the presence of $\mathrm{NaCl}$, and this effect has been attributed to a screening of polyelectrolyte charges, with the authors of this contribution stating that highly charged segments contribute to gel formation ${ }^{36}$. Mehta et al. also showed that $\mathrm{NaCl}$ can shield electrostatic interactions and impact the modulus of the gels. ${ }^{37}$ In the case in hand, in other words a weakening of the gel state in these elastin-based systems, further research is required in order to understand the underlying molecular phenomena that result in such changes in the rheological properties. However, the aforementioned studies induce us to consider that the interaction between the $\mathrm{NaCl}$ and the negative charges of the glutamic residues of the recombinamers (Table 1 and Table 2) may be responsible of the decrease in gel stability.

From a practical point of view, glucose $5 \%$ was selected as the dispersing medium for (EI)x 2 and (EIS)x2 since the stability of both systems (Table 5) is enhanced in this medium.

\subsection{In vitro drug release studies}

Clear difference can readily be seen in the drug-release profiles shown in Figure 3. Specifically, the (EIS)x2 system shows a more sustained release when compared to its (EI)x2 counterpart, with the percentage release after $8 \mathrm{~h}$ being $80.39 \%$ and $40.04 \% \mathrm{TM}$ for (EI)x2 and 
(EIS)x2, respectively. These data point to a relationship between the mechanical properties of the hydrogels and the rate of drug release. Thus, in concordance with its enhanced mechanical performance when compared to (EI)x2, (EIS)x2 shows the most sustained release.

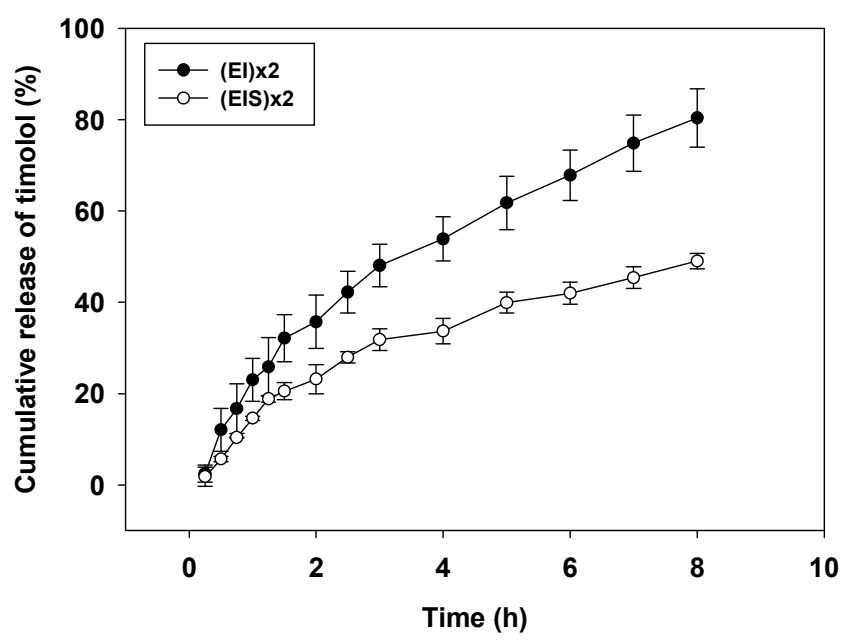

Figure 3: Release profiles of TM from (EI)x2 and (EIS)x2 hydrogels.

Drug delivery data were fitted to the Korsmeyer-Peppas model in order to further analyze the experimental results and to obtain quantitative information that could facilitate the comparison of both release profiles (Table 6).

The rate constant $\mathrm{K}$ was lower for the (EIS)x2 formulation than for its (EI)x2 counterpart, thus confirming a slower drug release by the former. The " $\mathrm{n}$ " value obtained for (EIS)x2 (n=0.58) and for $(\mathrm{EI}) \mathrm{xs}(\mathrm{n}=0.62)$ points to Fickian diffusion as the main mechanism governing the release. However, erosion processes are also involved in drug delivery as the values obtained are higher than 0.5 . Specifically, the " $n$ " value for (EI)x2 is higher than that for (EIS)x2, which points to a 
higher influence of erosion processes for (EI)x2. Consequently, erosion tests were performed to corroborate this (see next section).

Table 6: Values obtained after fitting the drug delivery profiles to the Korsmeyer-Peppas, Higuchi and Zero order kinetics models.

\begin{tabular}{|c|c|c|}
\hline Formulations & TM(EI)x2 & TM(EIS)x2 \\
\hline \multicolumn{3}{|c|}{ Korsmeyer-Peppas Model } \\
\hline $\mathrm{k}\left(\mathrm{hs}^{-\mathrm{n}}\right)$ & $22.685 \pm 0.973$ & $14.930 \pm 0.887$ \\
\hline $\mathrm{n}$ & $0.619 \pm 0.026$ & $0.588 \pm 0.036$ \\
\hline $\mathrm{R}^{2}$ & 0.988 & 0.973 \\
\hline \multicolumn{3}{|c|}{ Higuchi Model } \\
\hline $\mathrm{k}\left(\mathrm{hs}^{-1 / 2}\right)$ & $26.988 \pm 0.724$ & $16.965 \pm 0.469$ \\
\hline $\mathrm{R}^{2}$ & 0.962 & 0.958 \\
\hline \multicolumn{3}{|c|}{ Zero-OrderModel } \\
\hline $\mathrm{k}(\mathrm{hs})$ & $11.831 \pm 0.694$ & $7.390 \pm 0.496$ \\
\hline $\mathrm{R}^{2}$ & 0.824 & 0.764 \\
\hline
\end{tabular}

\subsection{In vitro erosion testing of the recombinamers}

Erosion tests were performed in order to determine possible differences in the stability between the two recombinamers. As shown in Figure 4, (EIS)x2 displays a significantly lower erosion than (EI)x2. This is in agreement with the presence of the SL motif in (EIS)x2 as said motif has been reported to provide enhanced stability against erosion in an excess of aqueous media in in vitro tests. This performance is likely to be translated into an increase in the residence of ophthalmic formulations containing (EIS)X2. Consequently, the next set of experiments was performed in vivo to test this hypothesis. 


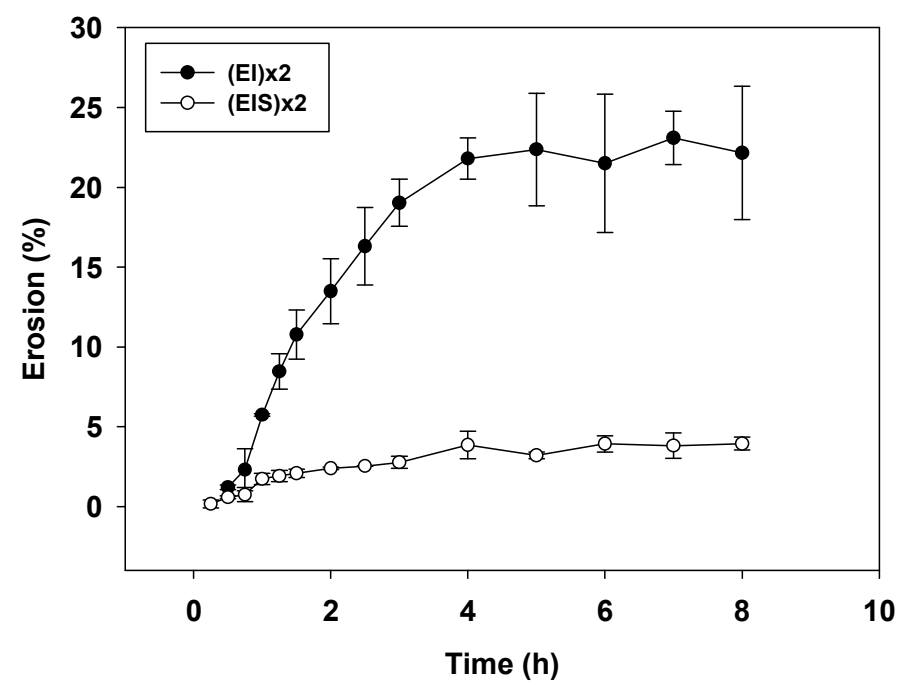

Figure 4: Erosion profiles of (EI)x2 and (EIS) x2 at 15 wt.\%.

\subsection{Cytocompatibility}

Cytocompatibility assays were performed in order to check the suitability of these recombinamers for biomedical applications. A fibroblast cell line (HFF-1) was used as cell model to test the cytocompatibility as fibroblasts are the predominant cell type in the ECM and, thereof, represent one of the main portals of exposure to biomaterials. A quantitative analysis was performed by measuring the corresponding fluorescence emitted by both calcein and EtDH1 under three test conditions, namely HFF-1 culture treated with i) (EI)x2, ii) (EIS)x2 or iii) without any recombinamer (control) for $24 \mathrm{~h}$, as detailed in the experimental section.

No statistically significant differences in cell viability were found between the treatment groups (Figure 5a) and microscopic observation of the cells corroborated these findings (Figure 5b-d). Furthermore, no morphological differences were observed between the fibroblasts treated 
a)

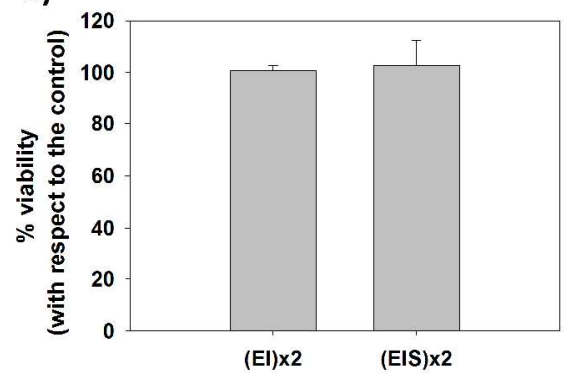

Recombinamer

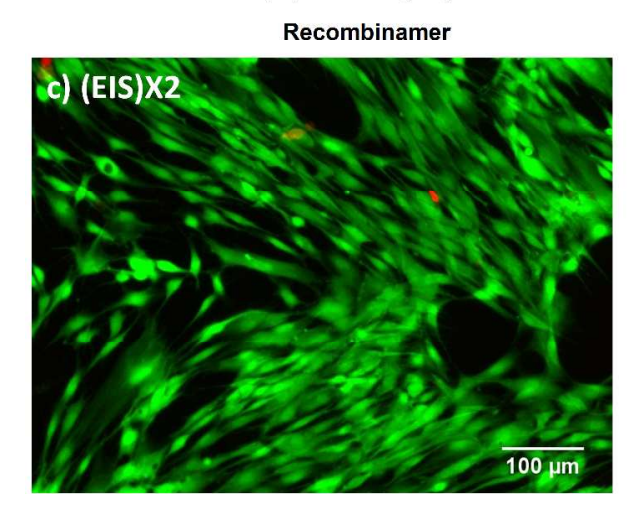

with the recombinamers and the control fibroblasts. As such, these results show the cytocompatible nature of these recombinamers and further support their potential application in the biomedical field.
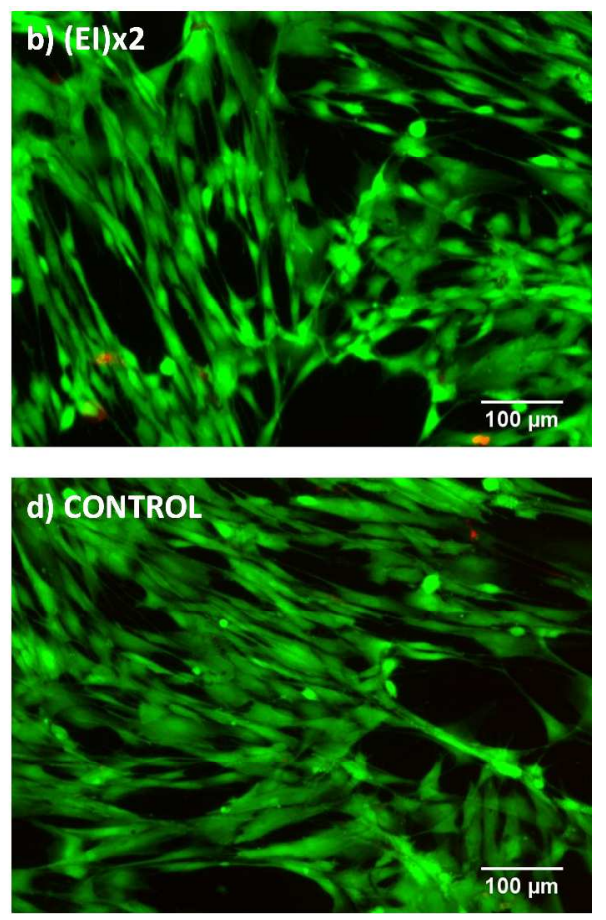

Figure 5: LIVE and DEAD differential staining of HFF-1 cells following 24 hours of TC-PS (Tissue Culture-Polystyrene Surface) culture in the presence of DMEN medium supplemented with the corresponding recombinamer a) Representation of the percentage of viability (with respect to the control) after $24 \mathrm{~h}$ of culture of HFF-1 cells in the presence of (EI)x2 and (EIS) x2. Three experiments were performed, each in triplicate. Data are expressed as mean \pm standard deviation. b) to d) Representative fluorescence microscopy images. Live cells appear in green whereas dead cells appear in red. 


\subsection{In vivo mucoadhesion tests}

Adhesion tests were performed in order to determine any differences in retention of the formulation on the eye surface that could potentially affect topical absorption of the drug.

Figure 6 shows that, immediately after instillation of the formulations $(\mathrm{t}=0)$, adhesion seems to be higher for (EIS)x2 than for (EI)x2, with both formulations presenting a higher score than the control, although the apparent differences are not statistically significant. This trend was maintained throughout the study, and between 5 and $15 \mathrm{~min}$, the differences between the three groups were statistically significant, with $\mathrm{p}<0.05$ in all cases. No statistically differences were detected between the control and the formulation containing (EI)x2 between 30 to $45 \mathrm{~min}$, whereas the (EIS)x2 formulation still presented statistically significantly higher adhesion properties, and this formulation could still be detected at $75 \mathrm{~min}$.

Thus, formulations containing either of the two recombinamers displayed better adhesion than the control. This is clearly important as regards the development of ophthalmic formulations since rapid washing-out and shear-thinning of mucoadhesive systems is a considerable obstacle that must be overcome when developing drug carriers to be administered in anatomical sites such as the ocular surface, where the clearance time for the tear film is $5-10 \mathrm{~s}^{38,39}$. Herein we show that the incorporation of either of these two recombinamers results in an increase in the residence time of the formulation on the eye surface. Moreover, differences were also detected between (EI)x2 and (EIS)x2, with the latter being more effective at increasing adhesion. This increase in adhesion can be related to the enhanced rheological properties and lower erosion displayed by the (EIS)x2 system when compared to (EI)x2 when $\mathrm{NaCl}$ is present, as is the case for the eye surface. 


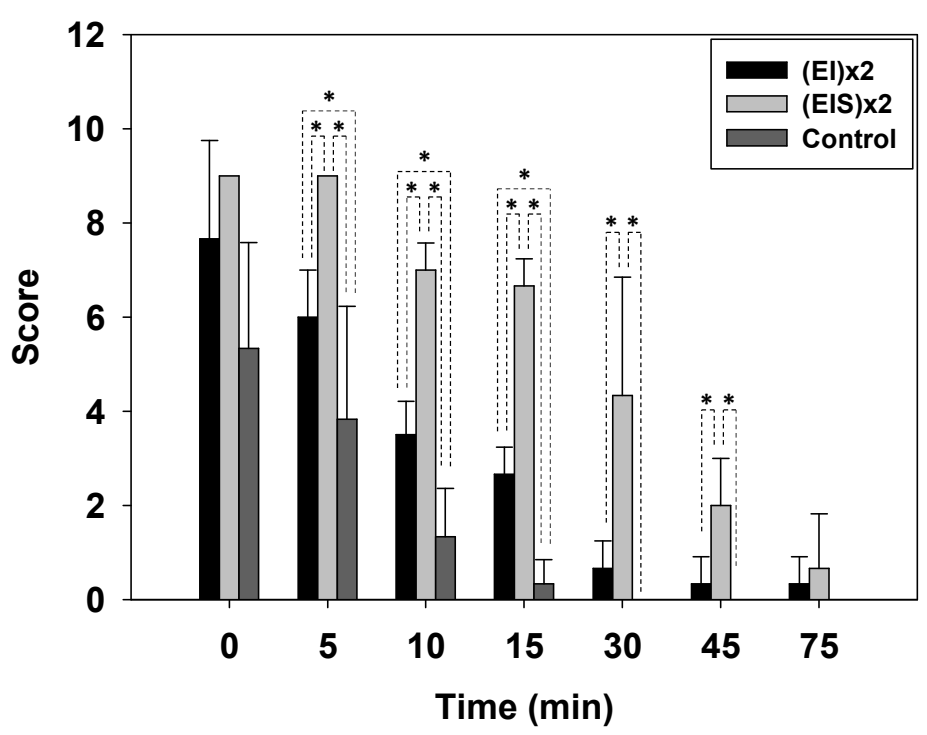

Figure 6: Adhesion score versus time for the recombinamer solutions and control (solution without recombinamer), $n=3$ rabbits. Data are expressed as mean \pm standard deviation. Statistical significance $(p<0.05)$ is marked with an asterisk.

\subsection{In vivo study of the hypotensive efficacy: IOP determinations}

As shown in Figure 7, formulations containing TM produced a decrease in the IOP, with no statistical differences being detected between them in the first three hours. However, this situation changed at $4 \mathrm{~h}$ post-administration, when the IOP for eyes treated with the formulation lacking recombinamer was statistically significantly higher than that displayed by the eyes treated with $(\mathrm{EIS}) \times 2(\mathrm{p}<0.05)$. From $4 \mathrm{~h}$ onwards, the TM solution presented no hypotensive effects, while those formulations containing $\mathrm{TM}$ and the respective recombinamer, namely (EI) $\mathrm{2}$ or (EIS) $\mathrm{x}$, maintained a reduced IOP, with this effect being more pronounced for the formulation containing (EIS)x2. In this case, the hypotensive effect lasted for more than $8 \mathrm{~h}$. 
These findings are in concordance with our initial hypothesis, in which we speculated that elimination of the drug as a result of lacrimal turnover may be minimized by the use of in situ gel-forming systems, thus leading to an enhanced hypotensive effect of the formulation. Furthermore, the differences encountered between (EI)x2- and (EIS)x2-containing systems agree with an enhanced stability of the latter under saline conditions. As $\mathrm{NaCl}$ constitutes one of the main components of lacrimal fluid ${ }^{40,41}$, it is expected to diffused over time into the gelformulation and decrease its mechanical properties, especially in the case of (EI)x2 (as shown in the rheological tests). Therefore, it is rational to suppose that the formulation containing (EIS)x2 displays a longer residence time than its counterpart containing (EI)x2, as was also demonstrated experimentally in the in vitro erosion tests and the in vivo adhesion tests, thus leading to an increased hypotensive effect. The results show that the formulation containing (EIS) 2 had a more sustained effect than a formulation containing just TM, in which the hypothesive effect lasted only $4 \mathrm{~h}$. Although hypotensive effects of up to $8 \mathrm{~h}$ are also displayed by commercially available preservative-containing formulations, such as Timoftol (FrosstLaboraties, Madrid, Spain) or Timolol Sandoz (Frosst Laboratories) ${ }^{42}$, it is important to note that the hypotensive effect achieved by the formulation containing (EIS) 2 was achieved without the use of preservatives. The inclusion of preservatives, such as benzalkonium chloride, ${ }^{43}$ is believed to favor TM penetration, and therefore its therapeutic efficacy, due to disruption of the hydrophobic barrier of the corneal epithelium. However, this adjuvant effect is produced at the expense of an increased toxicity and serious side-effects on the eye surface. ${ }^{44,} 45$ As such, preservative-free antiglaucoma eyedrops are believed to improve patient compliance and adherence in the medical treatment of this disease, and the introduction of preservative-free formulations that maintain efficacy is an important step towards the development of ophthalmic solutions ${ }^{46}$. Moreover, 
some studies have pointed to a possible role of the inclusion of polymers in the anti-glaucoma formulations in the reduction of ocular toxicity, thereby protecting the ocular surface in longterm therapies. ${ }^{42}$

The results reported herein support the feasibility of using (EIS)x2 as a component in a preservative-free anti-glaucoma formulation while maintaining the efficacy of the commercial benzalkonium chloride-containing versions. Moreover, the thermogelling behavior of this system allows easy self-administration, thus providing an advantage with respect to other polymeric systems in which pre-formed scaffolds are incorporated into the conjunctival sac, which can lead to patient discomfort. ${ }^{47,48}$

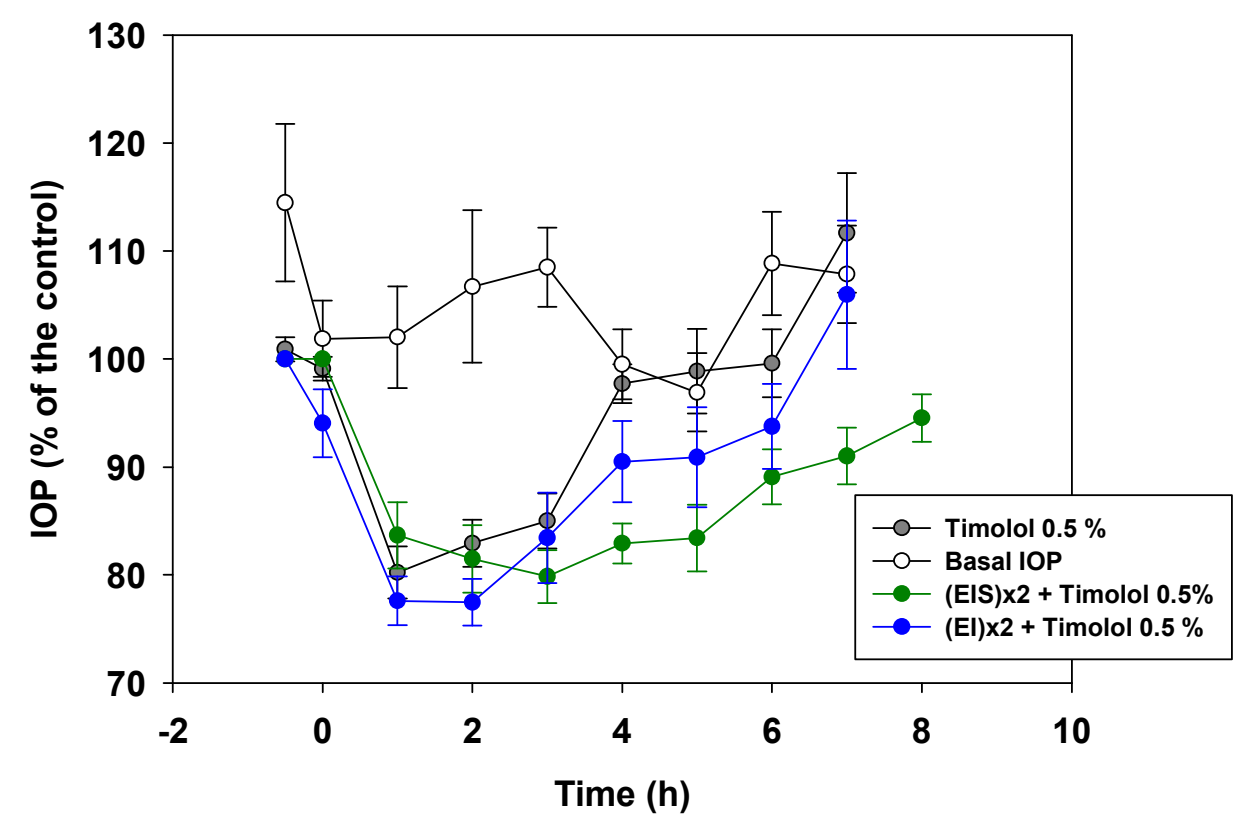

Figure 7: IOP evolution in normotensive New Zealand rabbits after administration of different TM formulations. Grey circles: IOP at different times after administration of TM $0.5 \%$ solution in glucose 5\% $(n=20$ eyes). White circles: basal IOP (glucose 5\% solution with no hypotensive 
agent) ( $n=10$ eyes). Green circles: IOP at different times after administration of the formulation containing TM $0.5 \%$ in (EIS)x2 at $15 \mathrm{wt} \%$ in glucose $5 \%$ ( $n=15$ eyes). Blue circles: IOP at different times after administration of the formulation containing TM 0.5\% in (EI) x2 at 15 wt \% in glucose $5 \%(n=15$ eyes $)$. Data are expressed as mean \pm standard error.

\subsection{In vivo ocular irritation test}

In order to evaluate the safety of the formulation containing (EI)x2 or (EIS)x2 $15 \mathrm{wt} \%$ with $\mathrm{TM}$ at $0.5 \%$ in dextrose solution when applied topically to rabbit eyes, irritation tests were performed as described in the experimental section. As shown in Table 7, no irritation was observed for either of the recombinamers as the score was less than 1 for all the times tested. Moreover, no significant differences were detected between the recombinamer formulations and the control group. The absence of irritation is in agreement with the reported biocompatible nature of this class of materials ${ }^{49}$, together with the aqueous base of the formulation and the lack of any preservatives, which maximizes the potential utility of these devices as drug-delivery systems.

Table 7: Irritation scores obtained for the two formulations tested, namely (EI) $x 2$ and (EIS) $x 2$ hydrogel at 15 wt.\% containing TM 0.5\% in the dispersing medium glucose 5\% and the commercial solution Zopirol 0.5\% TM as control. Three rabbits were used for each recombinamer formulation, with the right eyes being treated with the recombinamersolution and the left eyes being treated with the negative control. Data are expressed as mean \pm standard 


\begin{tabular}{|c|c|c|c|c|}
\hline & \multicolumn{4}{|c|}{ Time (min) } \\
\hline Sample & $\mathbf{3 0}$ & $\mathbf{6 0}$ & $\mathbf{9 0}$ & $\mathbf{1 2 0}$ \\
\hline $\mathbf{( E I ) x 2}$ & $0.33 \pm 0.58$ & $0.33 \pm 0.58$ & $1 \pm 0$ & $1 \pm 0$ \\
\hline $\mathbf{( E I S ) \times 2}$ & $0.67 \pm 0.58$ & $0 \pm 0$ & $0 \pm 0$ & $0 \pm 0$ \\
\hline Control & $0.50 \pm 0.55$ & $0.17 \pm 0.41$ & $0.33 \pm 0.51$ & $0.67 \pm 0.51$ \\
\hline
\end{tabular}

\section{CONCLUSIONS}

Although numerous studies have been conducted into the development of new anti-glaucoma formulations in order to reduce IOP to a greater extent, further research is still required. In this sense, the combination of biomaterials science and pharmacology is a must in order to find new solutions and approaches to overcome the current problems of ophthalmic drug delivery. Herein we have evaluated thermosensitive elastin and silk-elastin-like recombinamers as innovative pharmaceutical dosage forms for the topical administration of TM. Aqueous dispersions of recombinamers remained very fluid at low temperatures, which facilitated drug incorporation and administration. However, they were able to change into a gel form at physiological temperature so that TM could come entrapped inside the gel and experienced a sustained release. In vivo studies conducted in New Zealand rabbits showed that the incorporation of these recombinamers into a pharmaceutical formulation containing TM prolonged its retention on the preocular surface, leading to a greater decrease in the IOP. This effect was more evident in the case of the silk-elastin-like recombinamer (EIS)x2, which is in agreement with the enhanced 
stability of this material in the presence of a saline aqueous environment, as it is the scenario of the eye surface. Furthermore, these recombinamers can be placed in the eye without causing irritating effects or tear turnover, as demonstrated by the irritation tests, thereby maximizing the potential utility of these devices as drug-delivery systems. Therefore, (EIS)x2 constitute a novel and versatile type of hydrogel to address the critical issues that ophthalmic drug delivery entails.

In view of the above, and considering the potential offered by recombinant technology to develop further designs, the next step will focus on the development of a battery of recombinamers based on these initial designs, in order to further improve these outcomes. Specifically, different guest residues will be engineered in the amino acid sequence in order to further increase the retention in the pre-ocular surface, besides facilitating the handling of their aqueous solutions at room temperature.

\author{
ASSOCIATED CONTENT \\ Supporting Information. \\ The following files are available free of charge: DSC scans for $15 \mathrm{wt} \%$ (EIS)x2 and (EI)x2 \\ solutions (PDF)
}

\title{
AUTHOR INFORMATION
}

\section{Corresponding Authors}

* F. Javier Arias. Bioforge Lab, University of Valladolid, CIBER-BBN, Paseo de Belén 19, 47011 Valladolid, Spain. email: arias@bioforge.uva.es. Tel.+34-983-185855

* Santiago D. Palma. Unidad de Investigación y Desarrollo en Tecnología Farmacéutica 
(UNITEFA), CONICET and Departamento de Ciencias Farmacéuticas, Facultad de Ciencias Químicas, Universidad Nacional de Córdoba, Ciudad Universitaria, 5000-Córdoba, Argentina.,

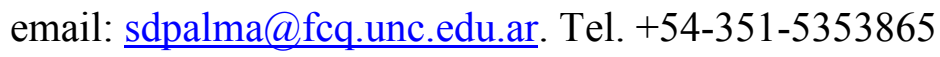

\section{Present Addresses \\ $\uparrow$ Department of Biohybrid \& Medical Textiles (BioTex) at AME-Institute of Applied Medical Engineering, Helmholtz Institute \& ITA-InstitutfürTextiltechnik, RWTH Aachen University, Pauwelsstr. 20, 52074. Aachen, Germany.}

\section{Author Contributions}

The manuscript was written through contributions of all authors. All authors have given approval to the final version of the manuscript. $\$$ These authors contributed equally.

\section{ACKNOWLEDGMENT}

The authors are grateful for the European Social Fund (ESF) and the European Regional Development Fund (ERDF) funding from the EU (NMP-2014-646075, HEALTH-F4-2011-278557, PITN-GA-2012-317306 and MSCA-ITN-2014-642687), the MINECO (MAT2015-68901-R, MAT2016-79435-R and MAT2016-78903-R), the JCyL (projects VA244U13 and VA313U14), the CIBER-BBN, the JCyL, the Instituto de Salud Carlos III under the "Network Center of Regenerative Medicine and Cellular Therapy of Castilla and Leon", the assistance of the "Consejo Nacional de InvestigacionesCientíficas y Técnicas (CONICET)” and Universidad Nacional de Córdoba. 


\begin{abstract}
ABBREVIATIONS
ELR, Elastin-like recombinamer; SELR, Silk-elastin like recombinamer; TM, timolol maleate, ITT, inverse temperature transition; ITC, inverse transition cycling; Tt, transition temperature; IOP, intraocular pressure.
\end{abstract}

\title{
REFERENCES
}

1. Quigley, H. A.; Broman, A. T. The number of people with glaucoma worldwide in 2010 and 2020. Br. J. Ophthalmol. 2006, 90, (3), 262-7.

2. Marquis, R. E.; Whitson, J. T. Management of glaucoma: focus on pharmacological therapy. Drugs Aging 2005, 22, (1), 1-21.

3. Schmidl, D.; Schmetterer, L.; Garhofer, G.; Popa-Cherecheanu, A. Pharmacotherapy of glaucoma. J. Ocul. Pharmacol. Ther. 2015, 31, (2), 63-77.

4. Nieminen, T.; Lehtimäki, T.; Mäenpää, J.; Ropo, A.; Uusitalo, H.; Kähönen, M. Ophthalmic timolol: Plasma concentration and systemic cardiopulmonary effects. Scand. J. Clin. Lab. Invest. 2007, 67, (2), 237-245.

5. Urtti, A. Challenges and obstacles of ocular pharmacokinetics and drug delivery. $A d v$. Drug. Deliv. Rev. 2006, 58, (11), 1131-5.

6. Knight, O. J.; Lawrence, S. D. Sustained drug delivery in glaucoma. Curr. Opin. Ophthalmol. 2014, 25, (2), 112-7.

7. Quinteros, D.; Vicario-de-la-Torre, M.; Andres-Guerrero, V.; Palma, S.; Allemandi, D.; Herrero-Vanrell, R.; Molina-Martinez, I. T. Hybrid formulations of liposomes and bioadhesive polymers improve the hypotensive effect of the melatonin analogue 5-MCA-NAT in rabbit eyes. PLoS ONE 2014, 9, (10), e110344.

8. Gratieri, T.; Gelfuso, G. M.; Rocha, E. M.; Sarmento, V. H.; de Freitas, O.; Lopez, R. F. A poloxamer/chitosan in situ forming gel with prolonged retention time for ocular delivery. Eur. J. Pharm. Biopharm. 2010, 75, (2), 186-93.

9. Fernandez-Colino, A.; Bermudez, J. M.; Arias, F. J.; Quinteros, D.; Gonzo, E. Development of a mechanism and an accurate and simple mathematical model for the description of drug release: Application to a relevant example of acetazolamide-controlled release from a bio-inspired elastin-based hydrogel. Mater Sci Eng C Mater Biol Appl 2016, 61, 286-92.

10. Altunbas, A.; Pochan, D., Peptide-Based and Polypeptide-Based Hydrogels for Drug Delivery and Tissue Engineering. In Peptide-Based Materials, Deming, T., Ed. Springer Berlin Heidelberg: 2012; Vol. 310, pp 135-167.

11. Zhao, X.; Zhang, S. Molecular designer self-assembling peptides. Chem. Soc. Rev. 2006, 35, (11), 1105-10.

12. Fernandez-Colino, A.; Arias, F. J.; Alonso, M.; Rodriguez-Cabello, J. C. Amphiphilic Elastin-Like Block Co-Recombinamers Containing Leucine Zippers: Cooperative Interplay 
between Both Domains Results in Injectable and Stable Hydrogels. Biomacromolecules 2015, 16, (10), 3389-98.

13. Rodríguez-Cabello, J. C.; Arias, F. J.; Rodrigo, M. A.; Girotti, A. Elastin-like polypeptides in drug delivery. Advanced Drug Delivery Reviews 2016, 97, (Supplement C), 85100.

14. Altman, G. H.; Diaz, F.; Jakuba, C.; Calabro, T.; Horan, R. L.; Chen, J.; Lu, H.; Richmond, J.; Kaplan, D. L. Silk-based biomaterials. Biomaterials 2003, 24, (3), 401-416.

15. Rodriguez-Cabello, J. C.; Girotti, A.; Ribeiro, A.; Arias, F. J., Synthesis of genetically engineered protein polymers (recombinamers) as an example of advanced self-assembled smart materials. In Nanotechnology in Regenerative Medicine, Navarro, M.; Planell, J. A., Eds. Humana Press: 2012; Vol. 811, p 319.

16. Rodriguez-Cabello, J. C.; Pierna, M.; Fernandez-Colino, A.; Garcia-Arevalo, C.; Arias, F. J. Recombinamers: combining molecular complexity with diverse bioactivities for advanced biomedical and biotechnological applications. Adv. Biochem. Eng. Biotechnol. 2011, 125, $145-$ 79.

17. Girotti, A.; Fernández-Colino, A.; López, I. M.; Rodríguez-Cabello, J. C.; Arias, F. J. Elastin-like recombinamers: Biosynthetic strategies and biotechnological applications. Biotechnol. J. 2011, 6, (10), 1174-1186.

18. Martin, L.; Javier Arias, F.; Alonso, M.; Garcia-Arevalo, C.; Rodriguez-Cabello, J. C. Rapid micropatterning by temperature-triggered reversible gelation of a recombinant smart elastin-like tetrablock-copolymer. Soft Matter 2010, 6, (6), 1121-1124.

19. Li, B.; Alonso, D. O. V.; Daggett, V. The molecular basis for the inverse temperature transition of elastin. J. Mol. Biol. 2001, 305, (3), 581-592.

20. Urry, D. W. Molecular Machines: How Motion and Other Functions of Living Organisms Can Result from Reversible Chemical Changes. Angew. Chem., Int. Ed. Engl. 1993, $32,(6), 819-841$.

21. Tamburro, A. M.; Guantieri, V.; Pandolfo, L.; Scopa, A. Synthetic fragments and analogues of elastin. II. Conformational studies. Biopolymers 1990, 29, (4-5), 855-870.

22. Fernandez-Colino, A.; Arias, F. J.; Alonso, M.; Rodriguez-Cabello, J. C. Self-organized ECM-mimetic model based on an amphiphilic multiblock silk-elastin-like corecombinamer with a concomitant dual physical gelation process. Biomacromolecules 2014, 15, (10), 3781-93.

23. Numata, K.; Kaplan, D. L. Silk-based delivery systems of bioactive molecules. $A d v$. Drug. Deliv. Rev. 2010, 62, (15), 1497-508.

24. Hardy, J. G.; Römer, L. M.; Scheibel, T. R. Polymeric materials based on silk proteins. Polymer 2008, 49, (20), 4309-4327.

25. Luo, Q.; Dong, Z.; Hou, C.; Liu, J. Protein-based supramolecular polymers: progress and prospect. Chem. Commun. (Camb) 2014, 50, (70), 9997-10007.

26. Klaikherd, A.; Nagamani, C.; Thayumanavan, S. Multi-stimuli sensitive amphiphilic block copolymer assemblies. J. Am. Chem. Soc. 2009, 131, (13), 4830-8.

27. Pinedo-Martín, G.; Santos, M.; Testera, A. M.; Alonso, M.; Rodríguez-Cabello, J. C. The effect of $\mathrm{NaCl}$ on the self-assembly of elastin-like block co-recombinamers: Tuning the size of micelles and vesicles. Polymer 2014, 55, (21), 5314-5321.

28. Pinedo-Martín, G.; Castro, E.; Martín, L.; Alonso, M.; Rodríguez-Cabello, J. C. Effect of Surfactants on the Self-Assembly of a Model Elastin-like Block Corecombinamer: From Micelles to an Aqueous Two-Phase System. Langmuir 2014, 30, (12), 3432-3440. 
29. Rodriguez-Cabello, J. C.; Girotti, A.; Ribeiro, A.; Arias, F. J. Synthesis of genetically engineered protein polymers (recombinamers) as an example of advanced self-assembled smart materials. Methods in molecular biology (Clifton, N.J.) 2012, 811, 17-38.

30. Ritger, P. L.; Peppas, N. A. A simple equation for description of solute release I. Fickian and non-fickian release from non-swellable devices in the form of slabs, spheres, cylinders or discs. J. Controlled Release 1987, 5, (1), 23-36.

31. Wilhelmus, K. R. The Draize eye test. Surv. Ophthalmol. 2001, 45, (6), 493-515.

32. Chambers, W. A.; Green, S.; Gupta, K. C.; Hill, R. N.; Huntley, K.; Hurley, P. M.; Lambert, L. A.; Lee, C. C.; Lee, J. K.; Liu, P. T.; et al. Scoring for eye irritation tests. Food Chem. Toxicol. 1993, 31, (2), 111-5.

33. Mehta, S. B.; Lewus, R.; Bee, J. S.; Randolph, T. W.; Carpenter, J. F. Gelation of a monoclonal antibody at the silicone oil-water interface and subsequent rupture of the interfacial gel results in aggregation and particle formation. Journal of pharmaceutical sciences 2015, 104, (4), 1282-90.

34. Tung, C.-Y. M.; Dynes, P. J. Relationship between viscoelastic properties and gelation in thermosetting systems. J. Appl. Polym. Sci. 1982, 27, (2), 569-574.

35. Reguera, J.; Urry, D. W.; Parker, T. M.; McPherson, D. T.; Rodriguez-Cabello, J. C. Effect of $\mathrm{NaCl}$ on the Exothermic and Endothermic Components of the Inverse Temperature Transition of a Model Elastin-like Polymer. Biomacromolecules 2007, 8, (2), 354-358.

36. Nowak, A. P.; Breedveld, V.; Pakstis, L.; Ozbas, B.; Pine, D. J.; Pochan, D.; Deming, T. J. Rapidly recovering hydrogel scaffolds from self-assembling diblock copolypeptide amphiphiles. Nature 2002, 417, (6887), 424-8.

37. Mehta, S. B.; Carpenter, J. F.; Randolph, T. W. Colloidal Instability Fosters Agglomeration of Subvisible Particles Created by Rupture of Gels of a Monoclonal Antibody Formed at Silicone Oil-Water Interfaces. Journal of pharmaceutical sciences 2016, 105, (8), 2338-48.

38. Sosnik, A.; das Neves, J.; Sarmento, B. Mucoadhesive polymers in the design of nanodrug delivery systems for administration by non-parenteral routes: A review. Prog. Polym. Sci. 2014, 39, (12), 2030-2075.

39. Greaves, J. L.; Wilson, C. G. Treatment of diseases of the eye with mucoadhesive delivery systems. Adv. Drug Delivery Rev. 1993, 11, (3), 349-383.

40. Murube, J.; Paterson, A.; Murube, E., Classification of Artificial Tears. In Lacrimal Gland, Tear Film, and Dry Eye Syndromes 2, Sullivan, D.; Dartt, D.; Meneray, M., Eds. Springer US: 1998; Vol. 438, pp 693-704.

41. Tiffany, J. M. Tears in health and disease. Eye 2003, 17, (8), 923-926.

42. Andres-Guerrero, V.; Vicario-de-la-Torre, M.; Molina-Martinez, I. T.; Benitez-delCastillo, J. M.; Garcia-Feijoo, J.; Herrero-Vanrell, R. Comparison of the in vitro tolerance and in vivo efficacy of traditional timolol maleate eye drops versus new formulations with bioadhesive polymers. Invest. Ophthalmol. Vis. Sci. 2011, 52, (6), 3548-56.

43. Noecker, R.; Miller, K. V. Benzalkonium chloride in glaucoma medications. Ocul. Surf. 2011, 9, (3), 159-62.

44. Pisella, P. J.; Pouliquen, P.; Baudouin, C. Prevalence of ocular symptoms and signs with preserved and preservative free glaucoma medication. Br. J. Ophthalmol. 2002, 86, (4), 418-23.

45. Jaenen, N.; Baudouin, C.; Pouliquen, P.; Manni, G.; Figueiredo, A.; Zeyen, T. Ocular symptoms and signs with preserved and preservative-free glaucoma medications. Eur. $J$. Ophthalmol. 2007, 17, (3), 341-9. 
46. Ishibashi, T.; Yokoi, N.; Kinoshita, S. Comparison of the short-term effects on the human corneal surface of topical timolol maleate with and without benzalkonium chloride. $J$. Glaucoma 2003, 12, (6), 486-90.

47. Gonzalez, A.; Tartara, L. I.; Palma, S. D.; Alvarez Igarzabal, C. I. Crosslinked soy protein films and their application as ophthalmic drug delivery system. Mater. Sci. Eng. C Mater. Biol. Appl. 2015, 51, 73-9.

48. Calles, J. A.; Tartara, L. I.; Lopez-Garcia, A.; Diebold, Y.; Palma, S. D.; Valles, E. M. Novel bioadhesive hyaluronan-itaconic acid crosslinked films for ocular therapy. Int. J. Pharm. 2013, 455, (1-2), 48-56.

49. Rincon, A. C.; Molina-Martinez, I. T.; de Las Heras, B.; Alonso, M.; Bailez, C.; Rodriguez-Cabello, J. C.; Herrero-Vanrell, R. Biocompatibility of elastin-like polymer poly(VPAVG) microparticles: in vitro and in vivo studies. J. Biomed. Mater. Res. A. 2006, 78, (2), 343-51. 
1

2

3

4

5

6

7

8

9

10

11

12

13

14

15

16

17

18

19

20

21

22

23

24

25

26

27

28

29

30

31

32

33

34

35

36

37

38

39

40

41

42

43

44

45

46

47

48

49

50

51

52

53

54

55

56

57

58

59

60

ACS Paragon Plus Environment 
For Table of Contents Use Only

Self-Assembling Elastin-Like Hydrogels for Timolol Delivery: Development of an Ophthalmic Formulation Against Glaucoma.

Alicia Fernández-Colino, $\$+\neq$ Daniela A. Quinteros, \# $\neq$ Daniel A. Allemandi, \# Alessandra Girotti, $\S$ Santiago D. Palma, \#*F. Javier Arias ${ }^{*}$.

† These authors contributed equally to this work.

§Bioforge Lab, University of Valladolid, CIBER-BBN, Paseo de Belén 19, 47011 Valladolid, Spain

\#Unidad de Investigación y Desarrollo en Tecnología Farmacéutica (UNITEFA), CONICET and Departamento de Ciencias Farmacéuticas, Facultad de Ciencias Químicas, Universidad Nacional de Córdoba, Ciudad Universitaria, 5000-Córdoba, Argentina.

\section{TOC/abstract graphic}

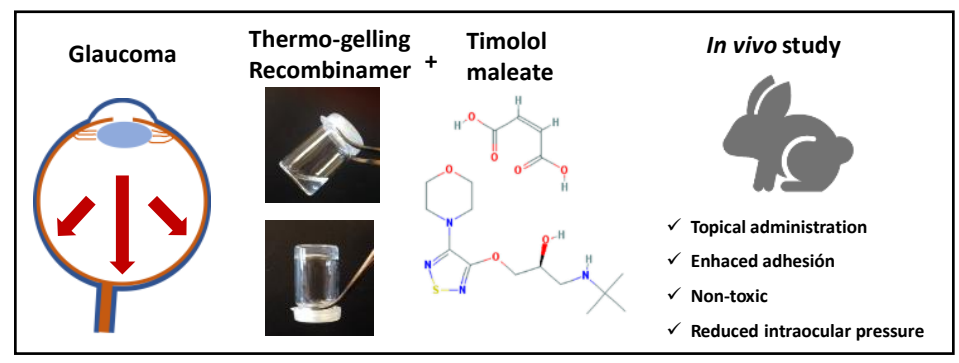

